\title{
EXPERIMENTAL BOVINE MASTITIS PRODUCED WITH HEMOLYTIC STREPTOCOCCI OF HUMAN ORIGIN *
}

\author{
David J. Davis and Joseph A. Capps \\ CHICAGO, ILL.
}

The Chicago epidemic of septic sore throat reported by Capps and Miller $^{1}$ was traced to a contaminated milk-supply at Batavia, Ill. It was found that on a number of farms supplying milk to Dairy $\mathrm{X}$, sore throat occurred in the milkers, and mastitis appeared in the cows; in some instances the sore throat appeared first, and in other instances the mastitis preceded the throat infections. These facts bring up the question whether or not it is possible for milkers suffering with sore throat to infect the teats or udders of cows by contaminated hands or otherwise during the process of milking. The question of the susceptibility of the cow to human streptococci is also involved. In an attempt to clear up these points some experiments were designed to test the pathogenicity of human streptococci for cows, and to determine possible avenues of udder infection through the teats.

For this purpose a healthy milch cow was obtained, giving at the time about 16 quarts of milk in twenty-four hours. Examination of the udder revealed no lesions, and examination of the milk from each quarter after proper cleansing of the teats showed no hemolytic streptococci on blood agar plates. Leukocytes in the milk from the quarters numbered from 10,000 to $30,000 .^{2}$

Experiment 1.-On three successive days the uninjured surfaces of the teats of the cow were smeared with milk cultures of typical hemolytic streptococci, recently isolated from cases of streptococcus tonsillitis. Two different strains were used. The result was entirely negative. Later, the teats were similarly smeared with a culture of streptococcus isolated during the milk epidemic from a fatal case of streptococcus infection. The result was also negative. A further experiment was made by smearing on the uninjured surface of the teat some grayish exudate teeming with streptococci which had just been removed from the tonsil of a severe case of tonsillitis. Examinations were made of both the first and last milk each day for a period of a week following these applications, but there was no evidence of infection. Streptococci were not found, and no increase in leukocytes appeared in any of the udder quarters.

* Received for publication February 9, 1914.

1. Jour. Am Med Assm. $1912,58,0.1848$.

2. The blood agar plate method was used in all the bacteriological examinations reported in this paper and the Doane-Buckley method was employed for counting leukocytes. 
Experiment 2.-A slight abrasion of the skin, sufficient to cause slight bleeding, was made at the end of one of the teats near the meatus. A culture of a hemolytic streptococcus was then directly applied. The wound became infected locally and there was slight swelling, redness, and evident tenderness. In the first milk some hemolytic streptococci appeared almost immediately, but they were practically absent from the last milk. After several days the streptococci appeared in large numbers and about the tenth day the letkocytes were markedly increased. For instance, in the first milk on the day following the infection the hemolytic streptococci in the milk from the involved quarter numbered 1,500 per c.c.; on the second day 5,000 , and on the third day 10,000 per c.c. They continued at about this number for some time and then became less. At the end of four weeks they were still present in numbers from 100 to 500 per c.c. Milk from the control quarter gave no streptococci. The leukocytes from the infected quarter, most of which were typical polynuclear cells, on the ninth day after inoculation numbered 250,000 per c.c., and on the twelfth day $1,408,000$ per c.c. For a time they remained about the same or slowly decreased, but at the end of one month they still numbered 100,000 per c.c. It is to be noted that at no time was the udder caked or swollen to any extent and milk obtained from this quarter was not "gargety" or stringy. From this experiment it appears that it is possible for the udder to be infected with human streptococci from an abrasion on the teat apparently by a direct infection ascending the canal.

Experiment 3.-In this experiment two twenty-four hour milk cultures of a hemolytic streptococcus, recently isolated from a typical case of ordinary follicular tonsillitis, were introduced into the udder by means of a catheter inserted to a distance of about $8 \mathrm{~cm}$. above the meatus. The amount of culture injected was 8 c.c. On the following day there was evidence of inflammation manifested by slight but distinct swelling of the quarter, some tenderness, and the presence of "gargety" milk. The quarter was not caked or hard. Enormous numbers of leukocytes were present and large numbers of typical hemolytic streptococci, especially in the first milk. They were present but were less numerous in the last milk. The streptococci continued to be present in the milk for several weeks and the marked increase in the number of leukocytes also continued for about the same length of time. Table 1 shows the number of streptococci and leukocytes per cubic centimeter from day to day in the milk from the infected quarter.

During the course of the experiments the cow remained in good physical condition. No appreciable increase of temperature was noted and there was no emaciation. The milk flow from the involved quarters was distinctly decreased during the infection but it later increased to the normal amount. The entire milk flow decreased during the course of the experiment rather more perhaps than one would expect under normal conditions. Lymph glands about the infected quarters were enlarged for a few days during the acute stage of the infection. There was no evidence that the infection spread from one quarter to another during the experiments. After about six weeks or two months, the teats and all the quarters of the udder became normal.

A point of interest in connection with these experiments is the possible effect that the milk might have on the properties of the streptococcus in the udder. This is important because it was noted that the streptococci isolated from many of the cases during the epidemic of sore throat had some rather characteristic properties which 
differentiated it from ordinary hemolytic streptococci. These were, briefly, its relative narrow zone of hemolysis, its moist mucoid growth, and the presence of a capsule. Careful observations were made of the streptococci from day to day as they were isolated from the milk on the blood agar plates. No change of properties whatever was noted. The streptococci, which were typical hemolytic streptococci when first isolated from the human throat, revealed no modification as to hemolysis, capsules, virulence, etc., even after growing in the udder for over four weeks. They still readily produced arthritis in rabbits.

TABLE 1.

The Number of Streptococci and Leukocytes in the Milk After Introduction of Streptococci in the Udder (Experiment 3 ).

\begin{tabular}{|c|c|c|}
\hline No. of Days After Injection of & $\begin{array}{l}\text { No. of Streptococci } \\
\text { per } 0.1 \text { c.c. }\end{array}$ & $\begin{array}{l}\text { No. of Leukocytes } \\
\text { per c.c. }\end{array}$ \\
\hline 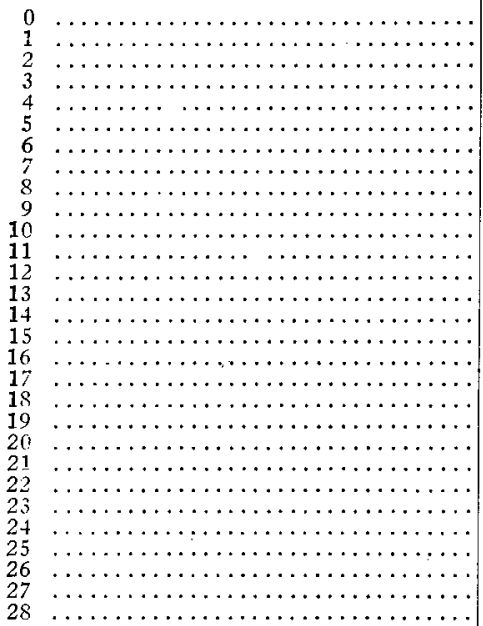 & $\begin{array}{r}0 \\
200 \\
500 \\
3,000 \\
1,200 \\
500 \\
150 \\
300 \\
1,500 \\
240 \\
175 \\
500 \\
200 \\
100 \\
400 \\
14 \\
20 \\
14 \\
\frac{-}{30} \\
\frac{11}{2} \\
\frac{2}{二} \\
0\end{array}$ & $\begin{array}{r}12,000 \\
9,840,000 \\
8,500,000 \\
5,200,000 \\
1,320,000 \\
\cdots 62 \ldots, 000 \\
624,000 \\
200,000 \\
275,000 \\
936,000 \\
472,000 \\
360,000 \\
320,000 \\
1,472,000 \\
512,000 \\
136,000 \\
448,000 \\
\overline{-} \\
9 \overline{44,000} \\
\overline{780,000} \\
\overline{260,000} \\
\overline{-} \\
\overline{190,000}\end{array}$ \\
\hline
\end{tabular}

Milk which came from the infected udder and contained many streptococci was fed in large amounts to rabbits, guinea-pigs and a monkey. After a period of from two to three weeks several guineapigs developed multiple arthritic lesions. In the affected joints there was swelling associated with hemorrhages. The rabbits and the monkey did not develop arthritis or other lesions. This point is now being more carefully investigated and feeding experiments with milk and with streptococci are being carried on to find out, if possible, 
what factors in the feeding experiments were responsible for the lesions in the guinea-pigs.

After carefully cleansing the meatus and removing the first milk it was possible to obtain a pure growth of streptococci from the infected udder. This was used as a basis to carry out some experiments to show the effect of temperature on the growth of these organisms in milk. The observations were made on the streptococci not only when growing pure in milk obtained directly from the udder, but also when growing in milk along with the ordinary contaminating bacteria.

TABLE 2.

The Effect of Refrigerator, Room and Incubator Temperatures on the Growth of Pure Streptococci in MilK

\begin{tabular}{|c|c|c|c|c|c|c|c|}
\hline Temperature & AtOnce & $\mathrm{r} / 2 \mathbf{H r}$. & $1 \mathrm{Hr}$. & 3 Hrs. & $6 \mathrm{Hrs}$. & $20 \mathrm{Hrs}$. & $48 \mathrm{Hrs}$. \\
\hline 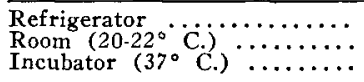 & $\begin{array}{l}1,100 \\
1,200 \\
1,200\end{array}$ & $\begin{array}{l}1,300 \\
1,200 \\
1,200\end{array}$ & $\begin{array}{l}1,300 \\
1,300 \\
1,300\end{array}$ & $\begin{array}{r}1,300 \\
1,300 \\
10,000\end{array}$ & $\begin{array}{r}1,400 \\
1,500 \\
20,000\end{array}$ & $\begin{array}{l}1,300 \\
7,000\end{array}$ & $\underline{1,000}$ \\
\hline
\end{tabular}

Table 2 shows the results obtained by permitting the milk containing pure streptococci to remain at refrigerator, room and incubator temperatures, plates being made at intervals in order to determine the number of organisms from time to time.

An examination of the table shows that the number of streptococi at refrigerator temperature remains practically the same for fortyeight hours. At room temperature $(20 \mathrm{C}$. $)$ there is some increase after six hours, and a decided increase at the end of twenty and forty-eight hours. In the incubator the multiplication of colonies is rapid, the number having increased tenfold after three hours. This result is about what one would expect with a pathogenic organism, such as the streptococcus.

TABLE 3.

The Effect of the Presence of Contaminating Bacteria on the Growth of Streptococci

\begin{tabular}{|c|c|c|c|c|c|c|c|}
\hline Temperature & At Once & T/2 Hr. & $1 \mathrm{Hr}$. & 3 Hrs. & $6 \mathrm{Hrs}$. & $22 \mathrm{Hrs}$. & $48 \mathrm{Hrs}$. \\
\hline 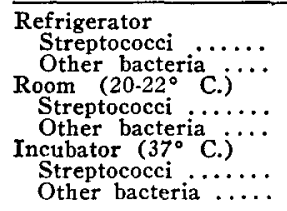 & $\begin{array}{r}39 \\
16 \\
92 \\
48 \\
46 \\
9\end{array}$ & $\begin{array}{r}64 \\
22 \\
40 \\
100 \\
78 \\
31\end{array}$ & $\begin{array}{r}45 \\
27 \\
79 \\
120 \\
67 \\
27\end{array}$ & $\begin{array}{r}55 \\
24 \\
97 \\
116 \\
106 \\
24\end{array}$ & $\begin{array}{r}67 \\
34 \\
66 \\
84 \\
\quad 111 \\
16\end{array}$ & $\begin{array}{r}72 \\
23 \\
260 \\
1,960 \\
3,200 \\
232\end{array}$ & $\begin{array}{c}80 \\
27 \\
250 \\
10,000+ \\
120 \\
10,000+\end{array}$ \\
\hline
\end{tabular}


Table 3 shows the effect of the presence of other bacteria on the growth of streptococci in mastitis milk. These bacteria are the ordinary contaminating bacteria found in milk when no special precautions are taken in the process of milking. They include the streptococcus lacticus, colon bacilli, etc., organisms which ordinarily are not pathogenic.

At refrigerator temperature, as one would expect, no appreciable increase of bacteria appears; at room temperature (20 to $22 \mathrm{C}$.) there is only a slight increase in the number of streptococci but a very great increase in the number of other germs. At incubator temperature the number of streptococci increase considerably in twentytwo hours, but at the end of forty-eight hours a marked diminution occurs. The other germs, however, show a marked increase at the end of forty-eight hours. Several similar experiments were made; there was noted some variation in the number of colonies both of streptococci and of other organisms at room temperature and at incubator temperature after twenty-four and forty-eight hours. This was due to the fact that the contaminating bacteria vary in number and kind and they may grow better at room temperature than at incubator temperature. However, in general, at the end of twenty-four and fortyeight hours there was a tendency for the contaminating bacteria to overgrow or inhibit the growth of the streptococci, as is readily seen by comparing Tables 1 and 2 . These data do not indicate that a brief exposure of milk containing streptococci to a favorable temperature, as might occur for example in the process of pasteurization, causes any appreciable increase in the number of bacteria.

In connection with these experiments, it should be stated that a number of tests showed that the streptococci in milk as it comes from the infected udder are readily killed by an exposure of twenty to thirty minutes at $60 \mathrm{C}$. $(140 \mathrm{~F}$.).

Of interest in connection with experimental mastitis is the onset, also the duration of the infection which, as we have seen, exists for several weeks at least. There is every reason to believe that the onset and duration of streptococcal mastitis as it occurs under natural conditions correspond to the onset and duration in experimental mastitis. These facts may be brought into relation with the onset and duration of the milk epidemics in which the outbreak is usually explosive in character and the duration usually continues for a number of weeks. The Chicago epidemic began suddenly about December 21, reached 
its maximum December 25, and then with the exception of a sudden rise on January 1, more or less gradually subsided, lasting about five or six weeks. The courses of the Boston and Baltimore epidemics corresponded in a general way to that of the Chicago epidemic, and the same may be said of the milk epidemics reported in England. It is seen, that there is a rather striking agreement between the onset and the duration of the experimental mastitis and the onset and duration of the milk-borne epidemics of septic sore throat.

\section{SUMMARY}

Hemolytic streptococci of human origin may cause mastitis, lasting for several weeks in cows. This time roughly corresponds to the duration of milk-borne epidemics.

The streptococci may gain entrance through an abraded or injured surface of the teat. It appears possible therefore for mastitis in cows to be produced by an infection from the milker whose hands are contaminated perhaps from a sore throat.

Mastitis results promptly when the cocci are injected directly into the udder by means of a catheter.

The mastitis may exist without physical evidence. A caked bag may not occur, though pus and streptococci in large numbers are being secreted in the milk.

Stringy, ropy, or gargety milk may or may not occur during the course of the mastitis.

In order to detect such infections, it might be necessary to examine milk from each quarter for bacteria, and for pus. This may explain the failure to detect the source of the streptococci in some of the epidemics of sore throat.

No change was noted in the cultural or pathogenic properties of the streptococci after growing in the udder of a cow for four weeks.

The presence of various contaminating bacteria tend to inhibit the growth of hemolytic streptococci in milk. 